\title{
Beurling algebras and uniform norms
}

\author{
by \\ S. J. Bhatt and H. V. Dedania (Vallabh Vidyanagar)
}

\begin{abstract}
Given a locally compact abelian group $G$ with a measurable weight $\omega$, it is shown that the Beurling algebra $L^{1}(G, \omega)$ admits either exactly one uniform norm or infinitely many uniform norms, and that $L^{1}(G, \omega)$ admits exactly one uniform norm iff it admits a minimum uniform norm.
\end{abstract}

A uniform norm on a Banach algebra $\mathcal{A}$ is a (not necessarily complete) algebra norm $|\cdot|$ satisfying the square property $\left|a^{2}\right|=|a|^{2}(a \in \mathcal{A})$. It is easy to see that any two equivalent uniform norms on a Banach algebra are identical. Thus two uniform norms are either identical or different. A Banach algebra $\mathcal{A}$ has the unique uniform norm property (UUNP) if it admits exactly one uniform norm; in this case, the spectral radius on $\mathcal{A}$ is the only uniform norm. Every regular, semisimple, commutative Banach algebra has UUNP; in particular, the uniform algebra $C(X)$ on a compact Hausdorff space $X$ and the group algebra $L^{1}(G)$ on a LCA group $G$ have UUNP. On the other hand, the disc algebra $\mathcal{A}(\mathcal{D})$ has infinitely many uniform norms [BhDe1]. Banach algebras with unique uniform norm have been investigated in [BhDe2], where it is shown that the Beurling algebra $L^{1}(G, \omega)$ (which is always semisimple [BhDe3]) has UUNP iff $L^{1}(G, \omega)$ is regular. In this paper we prove that $L^{1}(G, \omega)$ has either exactly one uniform norm or infinitely many uniform norms; and that it has UUNP if and only if it has a minimum uniform norm. This leads to the following question that remains open: Does there exist a (necessarily commutative and semisimple) Banach algebra $\mathcal{A}$ which admits finitely many distinct uniform norms?

Throughout let $G$ be a locally compact abelian (LCA) group, let $\lambda$ be a Haar measure, and let $\omega$ be a weight on $G$, i.e., a strictly positive measurable function $\omega$ on $G$ such that $\omega(s+t) \leq \omega(s) \omega(t)(s, t \in G)$. Then the Beurling algebra $L^{1}(G, \omega)$ consists of all complex-valued measurable functions $f$ on $G$

2000 Mathematics Subject Classification: 43A20, 46J05.

Key words and phrases: commutative semisimple Banach algebra, $\omega$-bounded generalized character, Beurling algebra. 
such that $f \omega \in L^{1}(G)$ is a semisimple commutative Banach algebra with the convolution product and the norm $\|f\|_{\omega}:=\int_{G}|f(s)| \omega(s) d \lambda(s)$ [BhDe3]. An $\omega$-bounded generalized character on $G$ is a continuous group homomorphism $\alpha: G \rightarrow(\mathbb{C} \backslash\{0\}, \times)$ such that $|\alpha(s)| \leq \omega(s)(s \in G)$. Let $H(G, \omega)$ denote the set of all $\omega$-bounded generalized characters on $G$ equipped with the compact-open topology. For $\alpha \in H(G, \omega)$, define

$$
\varphi_{\alpha}(f)=\widehat{f}(\alpha)=\int_{G} f(s) \alpha(s) d \lambda(s) \quad\left(f \in L^{1}(G, \omega)\right) .
$$

Then the map $T: H(G, \omega) \rightarrow \Delta\left(L^{1}(G, \omega)\right)$ defined as $T(\alpha)=\varphi_{\alpha}$ is a homeomorphism [BhDe4]. Let $\widehat{G}$ denote the dual group of $G$. Then it is easy to see that $\widehat{G} \subseteq H(G, \omega)$ iff $\omega \geq 1$ on $G$. However it is always true that $\theta \alpha \in H(G, \omega)(\theta \in \widehat{G}$ and $\alpha \in H(G, \omega))$. For $F \subseteq H(G, \omega)$, define

$$
|f|_{F}=\sup \{|\widehat{f}(\alpha)|: \alpha \in F\} \quad\left(f \in L^{1}(G, \omega)\right) .
$$

Then $|\cdot|_{F}$ is a uniform seminorm on $L^{1}(G, \omega)$; the set $F$ is a set of uniqueness for $L^{1}(G, \omega)$ if $|\cdot|_{F}$ is a norm. For example, $\alpha \widehat{G}$ is a set of uniqueness for any $\alpha \in H(G, \omega)$. For $\alpha \in H(G, \omega)$, the uniform norm $|\cdot|_{\alpha \widehat{G}}$ on $L^{1}(G, \omega)$ will be denoted by $|\cdot|_{\alpha}$.

TheOREM 1. $L^{1}(G, \omega)$ has UUNP iff it has a minimum uniform norm.

Proof. If $L^{1}(G, \omega)$ has UUNP, then clearly it has a minimum uniform norm. Conversely, assume that it has a minimum uniform norm, say $|\cdot|_{0}$. Define $F=\left\{\alpha \in H(G, \omega): \varphi_{\alpha}\right.$ is $|\cdot|_{0}$-continuous $\}$. By taking the completion $\mathcal{A}$ of $\left(L^{1}(G, \omega),|\cdot|_{0}\right)$ and by using elementary Gelfand theory, one can see that for all $f \in L^{1}(G, \omega),|f|_{0}=|f|_{F}=r_{\mathcal{A}}(f)$, the spectral radius in $\mathcal{A}$. Suppose that we have proved $F=H(G, \omega)$. This implies that $|\cdot|_{0}=|\cdot|_{F}=$ the spectral radius on $L^{1}(G, \omega)$ and the result is proved. So we prove $F=$ $H(G, \omega)$ in the following two steps.

STEP 1. $F=\widehat{G} F=\{\theta \alpha: \theta \in \widehat{G}$ and $\alpha \in F\}$.

Fix $\theta \in \widehat{G}$. Define $|f|_{\theta F}=|\theta f|_{F}$ on $L^{1}(G, \omega)$. Then $|\cdot|_{\theta F}$ is a uniform norm on $L^{1}(G, \omega)$. Since $|\cdot|_{F}\left(=|\cdot|_{0}\right)$ is the minimum uniform norm on $L^{1}(G, \omega)$, we have

$$
|f|_{F} \leq|f|_{\theta F} \quad\left(f \in L^{1}(G, \omega)\right) .
$$

This holds for each $\theta \in \widehat{G}$. So for each $\theta \in \widehat{G}$ and $f \in L^{1}(G, \omega)$, we have $|f|_{F} \leq|f|_{\theta F}$, i.e., $|f|_{F} \leq|\theta f|_{F}$. The last inequality is true for each $f$. So replacing $f$ by $\bar{\theta} f$, we get $|f|_{\bar{\theta} F}=|\bar{\theta} f|_{F} \leq|f|_{F}$. Thus for each $\alpha \in F$, the complex homomorphism $\varphi_{\bar{\theta} \alpha}$ is $|\cdot|_{0}$-continuous, i.e., $\bar{\theta} F \subseteq F$. This is true for each $\theta \in \widehat{G}$. Hence $F=\widehat{G} F$.

SteP 2. $F=H(G, \omega)$. 
Suppose, if possible, $F \neq H(G, \omega)$. Choose $\alpha_{1} \in F$ and $\beta_{1} \in H(G, \omega) \backslash F$. Take $\alpha=\left|\alpha_{1}\right|$ and $\beta=\left|\beta_{1}\right|$. Then by Step $1, \alpha \in F$ and $\beta \in H(G, \omega) \backslash F$. Choose $t \in G$ such that $\beta(t)<\alpha(t)$. Let $U$ be an open neighbourhood of $t$ in $G$ such that its closure $\bar{U}$ is compact and $\beta(s)<\alpha(s)(s \in U)$. Take $f=\chi_{U} \in L^{1}(G, \omega)$, the characteristic function of $U$. Now

$$
\begin{aligned}
|f|_{\beta} & =\sup \{|\widehat{f}(\beta \theta)|: \theta \in \widehat{G}\}=\sup \left\{\left|\int_{G} f(s) \beta(s) \theta(s) d \lambda(s)\right|: \theta \in \widehat{G}\right\} \\
& \leq \sup \left\{\int_{U} \beta(s)|\theta(s)| d \lambda(s): \theta \in \widehat{G}\right\} \leq \int_{U} \beta(s) d \lambda(s) \\
& <\int_{U} \alpha(s) d \lambda(s) \leq \sup \{|\widehat{f}(\alpha \theta)|: \theta \in \widehat{G}\}=|f|_{\alpha} \leq|f|_{F} .
\end{aligned}
$$

Thus $|\cdot|_{\beta}$ is a uniform norm and $|f|_{\beta}<|f|_{F}$, which is a contradiction because the latter is the minimum uniform norm on $L^{1}(G, \omega)$. This proves Step 2 and the result is proved.

The above result is not true in arbitrary semisimple commutative Banach algebras. For example, let $G$ be a non-discrete LCA group. Then the measure algebra $M(G)$ has a minimum uniform norm, namely $|\mu|_{\infty}=$ $\sup \{|\widehat{\mu}(\theta)|: \theta \in \widehat{G}\} \quad(\mu \in M(G))$ [BhDe2, Corollary 6.3]. But it does not have UUNP [BhDe2, p. 233]. Notice that both the disc algebra $A(\mathcal{D})$ and its variant $A_{r}(\mathcal{D})=\left\{f \in C(\mathcal{D}): f\right.$ is analytic on $\left.E_{r}\right\}$ (where $0<r<1$ and $E_{r}=\{z: r<|z|<1\}$ ) admit infinitely many uniform norms. However, $A(\mathcal{D})$ does not admit a minimum uniform norm, whereas $A_{r}(\mathcal{D})$ admits a minimum uniform norm, viz., $|f|_{0}=\sup \{|f(z)|:|z| \leq r\}\left(f \in A_{r}(\mathcal{D})\right)$.

Theorem 2. $L^{1}(G, \omega)$ admits either exactly one uniform norm or infinitely many uniform norms.

Proof. Assume that $L^{1}(G, \omega)$ has more than one distinct uniform norm. If $G$ is compact, then $L^{1}(G, \omega) \cong L^{1}(G)$ does have UUNP. Thus $G$ must be non-compact, and so its dual group $\widehat{G}$ is not discrete. Now choose $\alpha \in$ $H(G, \omega)$ and define $\widetilde{\omega}(s)=\omega(s) /|\alpha(s)|(s \in G)$. Then $\widetilde{\omega}$ is a weight and $\widetilde{\omega} \geq 1$ on $G$. It is easy to see that the map $T: L^{1}(G, \omega) \rightarrow L^{1}(G, \widetilde{\omega})$ defined as $T(f)=\alpha f$ is an isometric algebra isomorphism. Hence we may assume that $\omega \geq 1$ on $G$. Therefore $\widehat{G} \subseteq H(G, \omega)$. Now we define

$$
H_{\mathrm{p}}(G, \omega)=\{\alpha \in H(G, \omega): \alpha \text { is strictly positive }\} .
$$

As in the proof of Theorem 1, any two distinct elements $\alpha$ and $\beta$ in $H_{\mathrm{p}}(G, \omega)$ will give distinct uniform norms on $L^{1}(G, \omega)$, namely $|\cdot|_{\alpha}$ and $|\cdot|_{\beta}$. Now consider the following two cases:

CASE (i): $H_{\mathrm{p}}(G, \omega)$ is not a singleton. Choose $\alpha$ and $\beta$ in $H_{\mathrm{p}}(G, \omega)$ such that $\alpha \neq \beta$. Take $0<\lambda<1$. Define $\eta_{\lambda}(s)=\alpha(s)^{\lambda} \beta(s)^{1-\lambda}(s \in G)$. Then 
each $\eta_{\lambda} \in H_{\mathrm{p}}(G, \omega)$. Now if $0<\lambda \neq \lambda^{\prime}<1$, then $|\cdot|_{\eta_{\lambda}}$ and $|\cdot|_{\eta_{\lambda^{\prime}}}$ are distinct uniform norms on $L^{1}(G, \omega)$. Thus $L^{1}(G, \omega)$ admits infinitely many uniform norms.

CASE (ii): $H_{\mathrm{p}}(G, \omega)$ is a singleton. In this case $H_{\mathrm{p}}(G, \omega)=\left\{1_{G}\right\}$, where $1_{G}$ is the identity of $\widehat{G}$, and $H(G, \omega)=\widehat{G}$. Since $L^{1}(G, \omega)$ does not have UUNP, there exists a proper closed subset $F$ of $\widehat{G}$ which is a set of uniqueness for $L^{1}(G, \omega)$. Since $\widehat{G}$ is not discrete, $\widehat{G} \backslash F$ is infinite. Choose $\gamma_{1}, \gamma_{2}, \ldots$ to be different elements outside $F$. Set $F_{0}=F$ and $F_{n}=F_{n-1} \cup\left\{\gamma_{n}\right\}$. For each $n \geq 0$, define $|f|_{n}:=\sup \left\{|\widehat{f}(\gamma)|: \gamma \in F_{n}\right\}\left(f \in L^{1}(G, \omega)\right)$. Then each $|\cdot|_{n}$ is a uniform norm on $L^{1}(G, \omega)$. We show that they are distinct. It is enough to show that $|g|_{0}<|g|_{1}$ for some $g \in L^{1}(G, \omega)$. Since $L^{1}(G)$ is regular, there exists $f \in L^{1}(G)$ such that $\widehat{f}(F)=\{0\}$ and $\widehat{f}\left(\gamma_{1}\right)=1$. Fix $0<\varepsilon<1 / 2$. Then there exists $g \in C_{\mathrm{c}}(G)$ such that $\|f-g\|<\varepsilon$, where $\|\cdot\|$ is the $L^{1}$-norm on $L^{1}(G)$. Then $g \in C_{\mathrm{c}}(G) \subset L^{1}(G, \omega)$. Also

$$
|\widehat{g}(\gamma)|=|\widehat{g}(\gamma)-\widehat{f}(\gamma)| \leq\|\widehat{f}-\widehat{g}\|_{\widehat{G}} \leq\|f-g\|<\varepsilon<1 / 2 \quad(\gamma \in F) .
$$

Moreover,

$$
\begin{aligned}
\left|\widehat{g}\left(\gamma_{1}\right)\right| & =\left|\widehat{f}\left(\gamma_{1}\right)-\widehat{f}\left(\gamma_{1}\right)+\widehat{g}\left(\gamma_{1}\right)\right| \geq\left|\widehat{f}\left(\gamma_{1}\right)\right|-\left|\widehat{f}\left(\gamma_{1}\right)-\widehat{g}\left(\gamma_{1}\right)\right| \\
& \geq 1-\|f-g\|>1 / 2 .
\end{aligned}
$$

Thus $|g|_{0} \leq 1 / 2<|g|_{1}$. This completes the proof.

Define $\omega_{1}(s)=\exp (|s|)$ and $\omega_{2}(s)=(1+|s|)^{1 / 2}$ on $\mathbb{R}$. By $[\mathrm{D}$, Theorem 4.7.33], the Gelfand space of $L^{1}\left(\mathbb{R}, \omega_{1}\right)$ can be identified with the vertical strip $\Pi_{-1,1}:=\{x+i y:-1 \leq x \leq 1\}$ in the complex plane. For $-1 \leq x \leq 1$, define $|f|_{x}=\sup \{|\widehat{f}(x+i y)|: y \in \mathbb{R}\}\left(f \in L^{1}\left(\mathbb{R}, \omega_{1}\right)\right)$. Then each $|\cdot|_{x}$ is a uniform norm on $L^{1}\left(\mathbb{R}, \omega_{1}\right)$. By the maximum modulus principle, all of them are distinct norms. On the other hand, $L^{1}\left(\mathbb{R}, \omega_{2}\right)$ has exactly one uniform norm because it is regular [D, Theorem 4.3.37].

Remark. Assume that $\omega \geq 1$ on $G$. It follows from [BhDe2, Theorem 4.1] and [Do] that $L^{1}(G, \omega)$ has UUNP if and only if $\omega$ is non-quasianalytic, i.e., $\sum_{n \geq 1}(\log \omega(n s)) /\left(1+n^{2}\right)<\infty$ for each $s \in G$.

\section{References}

[BhDe1] S. J. Bhatt and H. V. Dedania, Banach algebras with unique uniform norm, Proc. Amer. Math. Soc. 124 (1996), 579-584.

[BhDe2] -, -, Banach algebras with unique uniform norm II, Studia Math. 147 (2001), 211-235.

[BhDe3] -, - A Beurling algebra is semisimple: an elementary proof, Bull. Austral. Math. Soc. 66 (2002), 93-95. 
[BhDe4] S. J. Bhatt and H. V. Dedania, Generalized characters, weighted measure algebras and uniform norms, submitted.

[D] H. G. Dales, Banach Algebras and Automatic Continuity, London Math. Soc. Monogr. 24, Clarendon Press, Oxford, 2000.

[Do] Y. Domar, Harmonic analysis based on certain commutative Banach algebras, Acta Math. 96 (1956), 1-66.

Department of Mathematics

Sardar Patel University

Vallabh Vidyanagar 388120

Gujarat, India

E-mail: subhashbhaib@yahoo.co.in

hvdedania@yahoo.com

Received September 12, 2002

Revised version April 23, 2003 